\title{
Conversion efficiency in silicon solar cells with spatially non-uniform doping
}

\author{
A.V. Sachenko, N.A. Prima, A.P. Gorban \\ Institute of Semiconductor Physics, NAS of Ukraine \\ 45, prospect Nauki, 252650 Kyiv, Ukraine
}

\begin{abstract}
The conversion efficiency of diffusion-type silicon solar cells, $\eta$, is studied theoretically in assumption of different doping levels existing under collection grid contacts and within the inter-contact spacing. It is shown that at high under-contact doping levels and at relatively low inter-contact doping ones the conversion efficiency increases as compared to uniform doping case. The dependence of $\eta$ on Shockley-Reed-Hall carrier lifetimes both in the base and in the top-surface $n^{+}$-layer as well as on the depth of $p$ - $n$-junction and the shape of electron concentration profile, $N(x)$, in the $n^{+}$-region is analysed.
\end{abstract}

Keywords: silicon solar cell, $p$ - $n$-junction, conversion efficiency.

Paper received 02.07.99; revised manuscript received 25.10.99; accepted for publication 26.10.99.

\section{Introduction}

As shown in papers [1-3], extremely high values of conversion efficiency in diffusion-type $n^{+}-p-p^{+}$silicon solar cells (SC) can be achieved only at high doping levels of $n^{+}$- and $p^{+}$-regions and at small thickness of top-surface $n^{+}$-layer $\left(\leq 10^{-5} \mathrm{~cm}^{-3}\right)$. That is caused by the necessity of minimization of SC internal resistance, effective surface recombination rates under top and rear surface contacts and recombination losses resulting from bulk recombination in the highly doped $n^{+}$- region. Important part of energy losses in SC is caused by short-circuit current reduction due to recombination of minority carriers and due to absorption of photoactive part of sun-light flux by free charge carriers within highly doped $n^{+}$- and $p^{+}$- regions. Besides, as it was shown in [4], conversion efficiency in diffusion-type silicon SC depends essentially on the maximum value of doping impurity concentration in the $n^{+}$-region and on the shape of $N(x)$ profile.

It is possible to decrease essentially energy losses caused by bulk and surface recombination as well as by free carrier absorption if under-contact semiconductor regions are doped to more high level than contact-free parts of SC surface. This conclusion was proved experimentally in [5], where essential enhancing of silicon SC performance due to nonuniform doping of under-contact and contact-free regions was observed. At the same time physical mechanisms of such enhancement are not studied theoretically in detail up to now. Our paper fills in this gap in the problem.

\section{Formulation of the problem}

The formulation of the problem is based mainly on approaches of papers [3, 4]. As in [3], the condition $d<L$ is supposed to be valid, where $d$ is SC thickness, and $L$ is the electron-hole pair diffusion length in the base. According to [4] it is suggested that in the region of $N(x)$ gradient the number of recombining electron-hole pairs is much less than that of generated.

Consider the case when the depth of $p$ - $n$-junction $x_{n}$ under contacts is equal to the summary thickness of the region $x_{n 0}$, where donor concentration is independent of coordinate $\mathrm{x}$ and of the region $\Delta x_{n}$, where donor concentration reduces as $x$ increases. As shown in $[6,7]$, such type of $N(x)$ profile are rather typical for diffusion-type silicon SCs and related to the presence of doping impurity solubility limit. Besides, in the varying part of $N(x)$ profile exponential dependences of the form $N_{0 c} \cdot \exp \left(\frac{-x}{x_{0}}\right)$ are often observed either in the whole $n^{+}$-region or in the essential part of it. The $p$ - $n$-junction depth $x_{n}$ in the above model is determined by the equation

$x_{n c}=x_{n 0}+x_{0} \ln \left(\frac{N_{0 c}}{p_{0}}\right)$,

where $N_{0 c}$ is the maximum value of donor concentration in the $n^{+}$-layer under contacts. 


\section{A.V. Sachenko et al.: Conversion efficiency in silicon solar cells with...}

Assume that in the inter-contact spaces classical diffusion profile of impurity concentration exists:

$N(x)=N_{0 i} \operatorname{erfc}\left(x / x_{1}\right)$.

Here, $N_{0 i}$ is the maximum value of donor concentration in the $n^{+}$-layer between contacts. The $p$ - $n$-junction depth $x_{n i}$ in this case is determined by the equation

$\operatorname{erfc}\left(x_{n i} / x_{1}\right)=p_{0} / N_{0 i}$.

The inequality $x_{n c}>x_{n i}$ is usually valid. $N(x)$ profiles illustrating concentration distribution under contacts and in the inter-contact spaces are shown in Fig.1.

The hole lifetime in highly doped $n^{+}$- region, according to [4], is assumed to be equal

$\tau_{p}=\left(\frac{1}{\tau_{r p}}+C_{n} N(x)^{2}\right)^{-1}$,

where $\tau_{r p}$ is Shockley-Reed-Hall lifetime of holes in the $n^{+}$-region, $C_{n}=2.8 \cdot 10^{-31} \mathrm{~cm}^{6} \cdot \mathrm{s}^{-1}$ is Auger recombination constant for electrons in silicon [8].

In calculations the following recombination channels in the base are taken into account: Shockley-Reed-Hall recombination, photon-emitting band-to-band recombination and Auger band-to-band recombination. In the used approximation $d<L$, the excess electron concentration in the base $\Delta n(x) \cong$ const, and for the bulk recombination rate, $R_{v}$, the following equation is valid:

$$
\begin{aligned}
R_{v} & =\Delta n \cdot d \cdot \mid \tau_{r n}^{-1}+A_{i}\left(p_{0}+\Delta n\right)+ \\
& \left.+C_{p}\left(p_{0}+\Delta n\right)^{2}+C_{n}\left(p_{0}+\Delta n\right) \Delta n\right]
\end{aligned}
$$

where $\tau_{r n}$ is the Shockley-Reed-Hall recombination lifetime, $A_{i}=1.48 .10^{-15} \mathrm{~cm}^{3} \mathrm{~s}^{-1}$ is the photon-emitting recombination constant at $T=300 \mathrm{~K}$ [9], $C_{p}=10^{-31} \mathrm{~cm}^{6} \mathrm{~s}^{-1}$ is the Auger recombination constant for holes [8]. Since recombination in the region of $N(x)$ gradient is supposed to be small, the influence of the mentioned region on the effective rate of surface recombination, $S_{0}$, at the front surface can be neglected. The corresponding criterion for this is the inequality

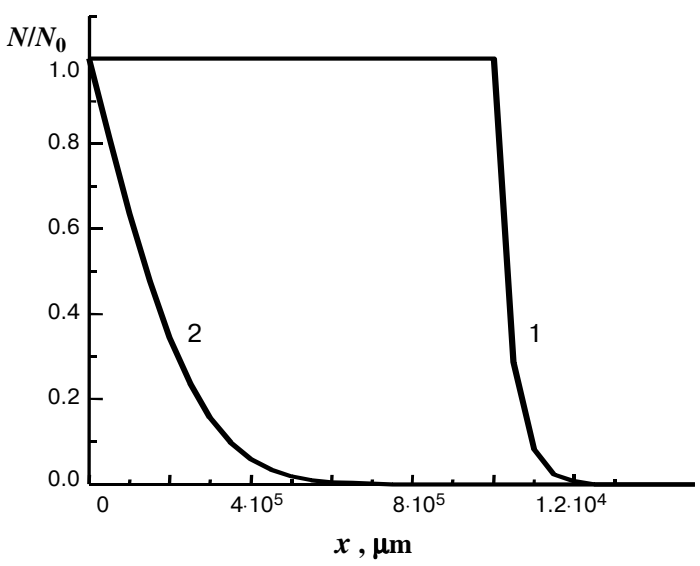

Fig.1. Dependence of electron concentration in the $n^{+}$-region under contacts (curve 1) and between contacts (curve 2) on coordinate $x$ : $N_{0 c}=10^{21} \mathrm{~cm}^{-3}, N_{0 i}=10^{19} \mathrm{~cm}^{-3}$
$E(x)=\frac{k T}{q} \frac{d N / d x}{N(x)}>>\frac{k T}{q L_{p}}$,

where $E(x)$ is the electric field strength, appearance of which is related to the presence of impurity gradient, $k T / q L_{p}$ is the diffusion-induced electric field strength in a given region, $L_{p}$ is the local diffusion length for holes. In this case recombination under contacts is determined not by a total $p-n$ junction depth $x_{n c}$, but only by $x_{n 0}$ region. Besides, recombination in the inter-contact spaces does not depend on $p$ - $n$ junction depth $x_{n i}$ and can be determined in frames of the diode theory for current flow. The effective recombination rate at the front surface, $S_{0}$, with account of the above approximations can be written as follows [3]:

$$
\begin{aligned}
S_{0}= & r_{0} \cdot\left(1+\frac{\Delta n}{p_{0}}\right), \\
r_{0}= & \frac{p_{0}}{N_{c}}\left\{V_{p} \exp \left(\Delta E_{n c}-Z_{n c}\right) \times\right. \\
& \times \frac{m\left(S_{p m} \cosh \left(\frac{x_{n 0}}{L_{p}}\right)+V_{p} \sinh \left(\frac{x_{n 0}}{L_{p}}\right)\right)}{}+ \\
+ & (1-m) S_{p m} \sinh \left(\frac{x_{n 0}}{L_{p}}\right)+V_{p} \operatorname{cosp}\left(\frac{x_{n 0}}{L_{p}}\right)
\end{aligned}
$$

where $V_{p}=D_{p} / L_{p}, D_{p}, L_{p}$ are, respectively, diffusion velocity, diffusion coefficient and diffusion length in highly doped $n^{+}$-region, $N_{c}$ is the effective density of states in the silicon conduction band, $\Delta E_{n c}$ and $\Delta E_{n i}$ reflect the reduction of silicon band-gap in the $n^{+}$- region (in $k T$ units) under and between the contacts, and magnitudes $Z_{n c}$ and $Z_{n i}$ are determined from equations

$F_{1 / 2}\left(Z_{n c}\right)=\frac{N_{0 c}}{N_{c}}, \quad F_{1 / 2}\left(Z_{n i}\right)=\frac{N_{0 i}}{N_{c}}$,

where $F_{1 / 2}(Z)$ is the Fermi-Dirac integral of the order of $1 / 2, S_{p m}$ and $S_{r}$ are «true» rates of surface recombination at the semiconductor-metal and semiconductor-dielectric interfaces, correspondingly, $m$ is the ratio of the top-contact grid area to the SC surface one.

For the effective recombination rate, $S_{d}$, at the rear surface in assumption of its total covering by metal we have similarly to (7), (8):

$$
\begin{aligned}
S_{d} & =r_{d} \cdot\left(1+\frac{\Delta n}{p_{0}}\right), \\
r_{d} & =V_{n} \frac{p_{0}}{N_{v}} \exp \left(\Delta E_{p}-Z_{p}\right) \times \\
\times & {\left[\frac{S_{n m} \cosh \left(\frac{x_{p 0}}{L_{n}}\right)+V_{n} \sinh \left(\frac{x_{p 0}}{L_{n}}\right)}{S_{n m} \sinh \left(\frac{x_{p 0}}{L_{n}}\right)+V_{n} \cosh \left(\frac{x_{p 0}}{L_{n}}\right)}\right], }
\end{aligned}
$$




\section{A.V. Sachenko et al.: Conversion efficiency in silicon solar cells with...}

where $V_{n}=D_{n}^{+} / L_{n}, D_{n}^{+}, L_{n}$ are, respectively, diffusion velocity, diffusion coefficient and diffusion length of electrons in $p^{+}$- region, $N_{v}$ is the effective density of states in the silicon valence band, $\Delta E_{p}$ is the reduction value of silicon band-gap in the $p^{+}$- region (in $k T$ units), and the magnitude $Z_{p}$ is determined from equation

$$
P_{0}=N_{v} \cdot F_{1 / 2}\left(Z_{p}\right) \text {, }
$$

where $P_{0}$ is the maximum value of hole concentration in $p^{+}$- region, $S_{n m}$ is the «true» surface recombination rate of electron-hole pairs in the semiconductor-metal interface, $x_{p 0}$ is the thickness of the region with $P(x)=$ const.

Parameters $S_{p m} \cong S_{n m} \cong 2,5 \cdot 10^{6} \mathrm{~cm} / \mathrm{s}$ were used in calculations, and it was assumed that the electron lifetime in $p^{+}$region is determined by band-to-band Auger recombination:

$$
\tau_{n^{+}}=\left(C_{p} \cdot P^{2}\right)^{-1} \text {. }
$$

The diffusion coefficient for holes in the $n^{+}$-region, $D_{p}$, was supposed to be $3.3 \mathrm{~cm}^{2} / \mathrm{s}$ [10], and the electron diffusion coefficient $D_{n}^{+}$in highly doped $p^{+}$- region - to $7 \mathrm{~cm}^{2} / \mathrm{s}$ [11]. For the dependences $\Delta E_{n}(N)$ and $\Delta E_{p}(P)$ the empirical relation from [12] was used:

$$
\begin{aligned}
& \Delta E_{n}(N)=0.0124 \cdot\left(\frac{N}{n_{i}}\right)^{0.25}, \\
& \Delta E_{p}(P)=0.0124 \cdot\left(\frac{P}{n_{i}}\right)^{0.25} .
\end{aligned}
$$

The magnitude of SC short-circuit current density in AM0 conditions (when the solar radiation spectrum is simulated by the radiation of absolutely black body at $T_{c}=5800 \mathrm{~K}$ ) at the ambient temperature $300 \mathrm{~K}$ is determined from the equation (in $\mathrm{A} / \mathrm{cm}^{2}$ ):

$$
J_{s c}=0.4505 \cdot(1-m) \cdot\left(1-r_{s}\right) \cdot \int_{0}^{1} \frac{f_{p}(\alpha(z))+f_{n}(\alpha(z))}{z^{4} \cdot\left[\exp \left(\frac{2.207}{z}\right)-1\right]} d z
$$

where $r_{s}$ is the reflection coefficient for the front SC surface, $z=\lambda \lambda_{x}, \lambda$ is the illumination wavelength, $\lambda_{x}$ is the red boundary of intrinsic photon absorption in silicon, $f_{p}(\alpha)$ and $f_{n}(\alpha)$ are the spectral dependences of collection coefficients for holes in the $n^{+}$- region and that for electrons in the $p$-region, $\alpha(z)$ is the spectral dependence of photon absorption coefficient the analytical expression of which is given in [13].

In accordance with the model [14], the function of electron-hole pair generation in semiconductor with taking into account multiple light reflection in the semiconductor is described by the expression:

$$
g(\alpha, x) \cong \alpha \cdot I \cdot \frac{\left(\exp (-\alpha x)+R_{d} \cdot \exp (-2 \alpha d+\alpha x)\right)}{1-R_{0} \cdot R_{d} \cdot \exp (-2 \alpha d)},
$$

where $I$ is the intensity of monochromatic illumination, $R_{0}$ and $R_{d}$ are reflection coefficients for photons moving to the top and rear SC surfaces from the semiconductor bulk. The situation $R_{0}=R_{d}=1$ models the case of total light absorption in the semiconductor that may occur for textured or profiled surfaces of SC.

The successive approximation method was used for determination of $f_{p}(\alpha)$. Since recombination is low in the region of $N(x)$ gradient, i.e. the inequality (6) is fulfilled, in the first approximation the recombination term can be neglected in the continuity equation for holes in the $n^{+}$region. This allows one to determine the distribution of nonequilibrium holes, $\Delta p(x)$, in this region. Taking into account, in the second approximation, the bulk recombination in the inter-contact spaces, and with account of $\Delta p(x)$ distribution we get the following expression for $f_{p}(\alpha)$ :

$$
\begin{aligned}
& f_{p}(\alpha)=1-\exp \left(-\alpha x_{n}\right)- \\
& -\frac{1}{D_{p}} \int_{0}^{x_{n}} \frac{\exp (-y(x))}{\tau_{p}(x)} \int_{x}^{x_{n}} \exp (y(t))(1-\exp (-\alpha t)) d t d x,
\end{aligned}
$$

where

$$
y(x)=\frac{2}{x_{1} \sqrt{\pi}} \int_{0}^{x} \frac{\exp \left(-\left(\frac{u}{x_{1}}\right)^{2}\right) d u}{1-\frac{2}{\sqrt{\pi}} \int_{0}^{u / x_{1}} \exp \left(-t^{2}\right) d t}
$$

is the dimensionless potential (normalized by $k T / q$ ) appearing in the $n^{+}$-region due to the gradient of concentration $N(x)$.

The magnitude $f_{n}(\alpha)$, in accordance with [4], is determined from equation

$$
\begin{aligned}
& f_{n}(\alpha) \cong-\frac{\alpha L}{\left(\alpha^{2} L^{2}-1\right) \cdot\left[1-R_{0} R_{d} \exp (-2 \alpha d)\right]} \times \\
& \times\left\{\left[\left(r_{d}+V\right) \cdot \exp \left(\frac{d-x_{n}}{L}\right)+\left(r_{d}-V\right) \cdot \exp \left(\frac{-d+x_{n}}{L}\right)\right] \times\right. \\
& \times\left[\exp \left(-\alpha x_{n}\right)+R_{d} \exp \left(-2 \alpha d+\alpha x_{n}\right)\right]+ \\
& \left.+2\left[\alpha D\left(1-R_{d}\right)-r_{d}\left(1+R_{d}\right)\right] \exp (-\alpha d)\right\} \times \\
& \times\left[\quad\left(r_{d}+V\right) \cdot \exp \left(\frac{d-x_{n}}{L}\right)-\left(r_{d}-V\right) \cdot \exp \left(\frac{d-x_{n}}{L}\right)\right]^{-1}+ \\
& +\frac{(\alpha L)^{2}\left[\exp \left(-\alpha x_{n}\right)-R_{d} \exp \left(-2 \alpha d+\alpha x_{n}\right)\right]}{\left(\alpha^{2} L^{2}-1\right) \cdot\left[1-R_{0} R_{d} \exp (-2 \alpha d)\right]},
\end{aligned}
$$

where $L$ and $V$ are diffusion length and diffusion velocity in the base.

Let us calculate now the conversion efficiency $\eta\left(N, x_{n}, P, x_{p}, p_{0}, d\right)$. Firstly, we determine the open cir- 


\section{A.V. Sachenko et al.: Conversion efficiency in silicon solar cells with...}

cuit voltage, $V_{O C}$, which is equal to the sum of voltage drops across top-surface and rear-surface regions under illumination:

$$
V_{0 C}=\frac{k T}{e} \cdot \ln \left[\frac{\Delta n}{n_{0}} \cdot\left(1+\frac{\Delta n}{p_{0}}\right)\right] .
$$

To find the excess carrier concentration, $\Delta n$, one should use the generation-recombination balance equation of the form:

$$
J_{S C} / e=R_{v}(\Delta n)+\left(S_{f}+S_{r}\right) \cdot \Delta n,
$$

where $J_{S C}$ is the short circuit current density.

It follows from (21) that in the open circuit mode the following relation is valid

$$
\Delta n=-\frac{p_{0}}{2}+\left[\frac{p_{0}^{2}}{4}+n_{i}^{2} \exp \left(\frac{e V_{0 C}}{k T}\right)\right]^{1 / 2} .
$$

Assuming, as usually, that forward bias and illumination (in the presence of loading resistor) give the equivalent result, SC I-V characteristic can be written as follows:

$$
\begin{aligned}
J(V) & =J_{S C}-e R_{v}\left(\Delta n^{*}\right)- \\
& -e S_{f}\left(\Delta n^{*}\right) \cdot \Delta n^{*}-e S_{r}\left(\Delta n^{*}\right) \cdot \Delta n^{*},
\end{aligned}
$$

where the magnitude $\Delta n^{*}$ is determined from (22) with $V_{0 C}$ substituted by $V$. Using the expression for maximum output power $P_{m}=J\left(V_{m} V_{m}\right.$, we get the transcendent equation for $V_{m}$ determination. The SC efficiency per unit area with account of results of [12] can be written as follows:

$\eta=\frac{J\left(V_{m}\right) V_{m}}{0.136} \frac{2 L_{c}}{l} \cdot \tanh \left(\frac{l}{2 L_{c}}\right)$,

where $L_{c}=\left(\frac{\mu_{n} M_{0} \cdot k T}{J_{s c}-J(V m)}\right)^{1 / 2}, M_{0} \cong \int_{0}^{x_{n}} N(x) d x, \mu_{n}-$ is the electron mobility in the $n^{+}$- region, $l$ is the distance between fingers of the contact grid.

Let us specify the geometry of the contact grid. We will consider it consisting of wide contact strip that connect the system and of narrow parallel fingers, so that the relative area of strip is $m_{1}$, the area of fingers is $m_{2}$ and their total area is $m$. In this case following simple relation between $m_{2}, l$ and finger width $l_{n}$ is valid:

$l=\frac{l_{n}\left(1-m_{2}\right)}{m_{2}}$.

\section{Discussion of results}

By use of above equations it is possible both to calculate the conversion efficiency $\eta$ for various sets of basic parameters of SC with spatially non-uniform doping and to study the problem of optimization of SC performance. In particular, we consider the dependences of $\eta$ on Shockley-ReedHall lifetimes in highly doped $n^{+}$-layer and in the base, on $p$ - $n$ junction depth, on doping levels under contacts, within the inter-contact spaces and in the base. In the next analysis we will use the following parameters of diffusion-type silicon SCs: $d=100 \mu \mathrm{m}, D_{n}=35 \mathrm{~cm}^{2} / \mathrm{s}, D_{p}=3.3 \mathrm{~cm}^{2} / \mathrm{s}$, $R_{0}=1, \quad R_{d}=1, m_{1}=0.025, r_{s}=0, x_{p 0}=10^{-4} \mathrm{~cm}$,

$P_{0}=5 \cdot 10^{20} \mathrm{~cm}^{-3}, \quad \mu_{n}=100 \frac{\mathrm{cm}^{2}}{\mathrm{~V} \cdot \mathrm{s}}, \quad l_{n}=0.0015 \mathrm{~cm}$, $S_{r}=10^{3} \mathrm{~cm} / \mathrm{s}$.

The variation of SC conversion efficiency with doping concentration in under-contact regions for spatially nonuniform (curves 1-3) and uniform (curve 4) cases is shown in Fig.2. While plotting the curve 4, photon absorption at free carriers in highly doped regions was taken into account similarly to [3]. It was assumed here that the quantum-mechanical theory of photon absorption developed in the Born approximation for the case of pulse scattering at ionized impurities [15] is valid, and the impurity compensation in $n^{+}$- and $p^{+}$-regions is absent. In last case calculation of the photon absorption coefficient can be done by modifying the equation for the generation function $g(\alpha, x)$. In particular, if $R_{0}=R_{d}=1$ (which corresponds to full absorption of radiation in SC) such relation is valid:

$g^{*}(\alpha) \cong \frac{\alpha I \sqrt{R_{1}}\left(e^{-\alpha x}+R_{2} e^{\alpha x-2 \alpha d}\right)}{1-R_{1} R_{2} e^{-2 \alpha d}}$,

where $R_{1}=\exp \left(-2 \alpha_{n} x_{n}\right), R_{2}=\exp \left(-2 \alpha_{p} x_{p}\right)$ are the light intensity reduction factors by $n^{+}$- and $p^{+}$-regions, and

$\alpha_{n}=1.8 \cdot 10^{-39} N^{2}(\lambda / \mathrm{mcm})^{3.5}$,

$\alpha_{p}=1.0 \cdot 10^{-39} P^{2}(\lambda / \mathrm{mcm})^{3.5}$

are the light absorption coefficients for electrons and holes, respectively.

As can be seen from Fig. 2, the conversion efficiency in the case of SC with a spatially non-uniform doping distri-

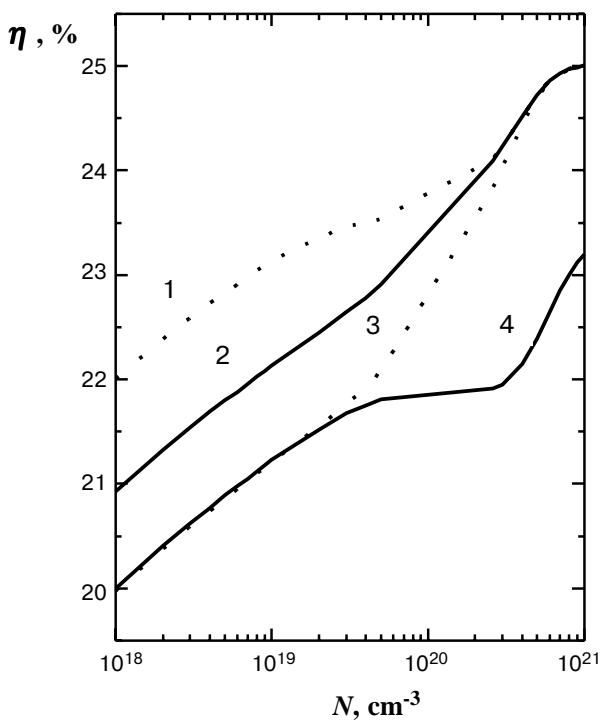

Fig.2. Dependencses of the conversion efficiency on maximum doping level of the $n^{+}$-region under contacts: $m=0.05, N_{0 i}=10^{18} \mathrm{~cm}^{-3}, x_{n i}=10^{-4} \mathrm{~cm}$, $x_{n c}=10^{-5}(1), 3 \cdot 10^{-5}(2), 10^{-4} \mathrm{~cm} \mathrm{(3),} \mathrm{curve} 4$ corresponds to the case of uniform doping at $m=0.05, S_{0}=S_{d}=0, x_{n}=3 \cdot 10^{-5} \mathrm{~cm}$. 


\section{A.V. Sachenko et al.: Conversion efficiency in silicon solar cells with...}

bution is higher than that for $\mathrm{SC}$ with uniform distribution at all values of $N_{0 c}$.

The dependences of $\eta$ on the doping concentration in the base are shown in Fig.3 with electron lifetime in the base, $\tau_{r n}$, as a parameter. Here electron and hole concentrations in highly doped regions are assumed to be constant.

As can be seen from the figure, the conversion efficiency increases essentially with $\tau_{r n}$ in the region of low base doping concentration, and at $\tau_{r n} \geq 10^{-2} \mathrm{~s}$ its magnitude becomes independent on the base doping level in a wide range of $p_{0}$ variation. The constancy of $\eta\left(p_{0}\right)$ is caused, on the one hand, by nonlinear excitation conditions in the base ( $\Delta n>p_{0}$ ) and, on the other hand, by small SC thickness resulting in participation of both top-surface and rearsurface space charge regions in photo-voltage formation. The increase in $\eta\left(p_{0}\right)$ as observed at $p_{0}>\Delta n$ is explained by the rise of photo-voltage with increase of potential barrier height. This increase is less pronounced if the contribution of Shockley-Reed-Hall recombination to the total recombination flux in the base is negligible.

In Fig. 4 the dependences of short-circuit current on $p$ - $n$ junction depth, $x_{n i}$, in the inter-contact spaces are plotted. The Shockley-Reed-Hall lifetime for holes, $\tau_{r p}$, is used as a parameter. As seen from the figure, maximum values of $J_{s c}$ at low $\tau_{r p}$ can be obtained by decreasing of $x_{n}$ values. At small $p$ - $n$ junction depths, $x_{n}$, the Ohmic losses of SC output power increase due to rise of sheet resistance of the $n^{+}$-region. To reduce these losses the spacing between parallel fingers of contact grid should be decreased. Besides, the degree of SC shadowing increases resulting in decrease of short-circuit current, and, hence, the $\eta$ value. The optimum is achieved at a proper relation between Ohmic losses and losses caused by grid shadowing of the top surface, as illustrated in Fig.5.

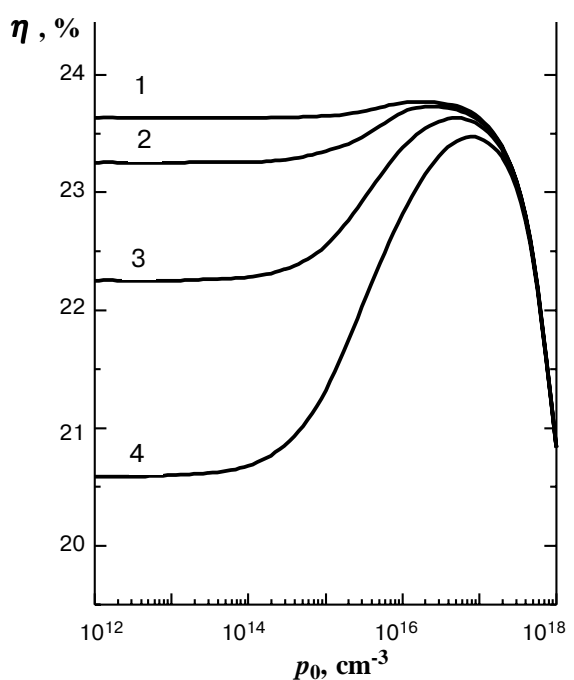

Fig.3. Dependences of the conversion efficiency on base doping level: $m=0.01, x_{n 0}=10^{-5} \mathrm{~cm}, N_{0 c}=10^{21} \mathrm{~cm}^{-3}, \tau_{r n}=3 \cdot 10^{-2}(1), 10^{-2}(2)$, $3 \cdot 10^{-3}(3), 10^{-3} \mathrm{~s}(4)$.

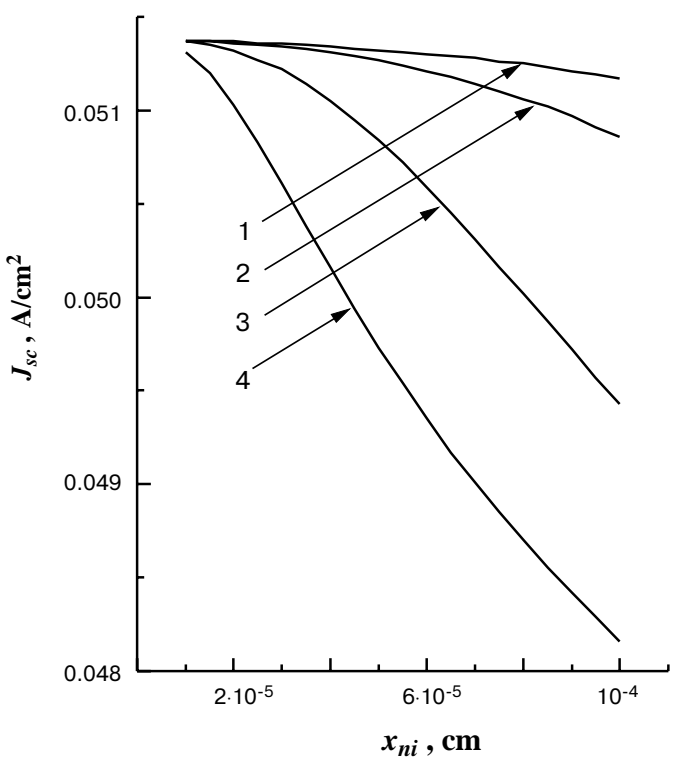

Fig.4. Dependences of the short-circuit current on $p$ - $n$ junction depth in the intercontact spaces: $m=0.05, N_{0 i}=10^{19} \mathrm{~cm}, S_{d}=0, \tau_{r p}=10^{-7}(1)$, $10^{-8}(2), 10^{-9}(3), 10^{-10} \mathrm{~s}(4)$.

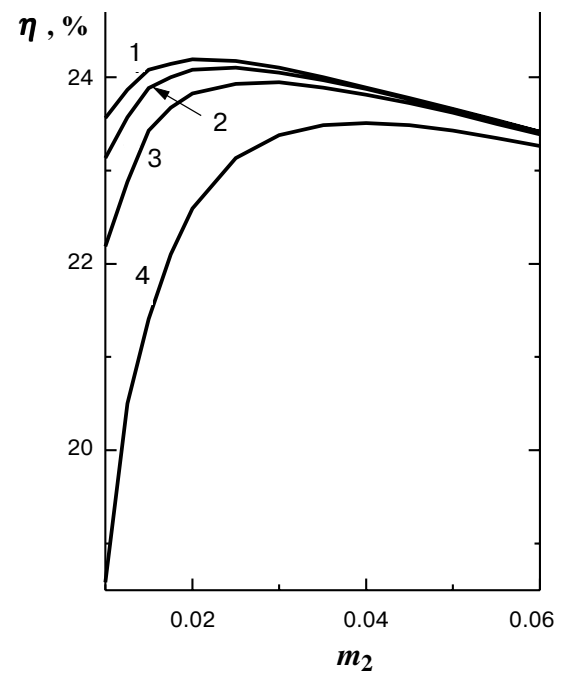

Fig.5. Dependences of the conversion efficiency on the shadowing of the front surface by parallel lines of the contact grid: $m_{1}=0.025, N_{c}=10^{21}$ $\mathrm{cm}^{-3}, x_{n 0}=10^{-4} \mathrm{~cm}, N_{i}=10^{19} \mathrm{~cm}^{-3}, S_{0}=S_{d}=0, x_{n i}=7 \cdot 10^{-5}(1), 5 \cdot 10^{-5}(2)$, $3 \cdot 10^{-5}(3), 10^{-5} \mathrm{~cm}(4)$.

\section{Conclusions}

It is shown that the conversion efficiency of diffusion-type $n^{+}-p-p^{+}$silicon SC increases with increase of doping concentration in the $n^{+}$-layer under top-surface contact grid in comparison with inter-contact spaces. As the ShockleyReed-Hall lifetime in the base, $\tau_{r n}$, increases, the conversion efficiency is enhanced essentially at low base doping levels, while at $\tau_{r n} \geq 10^{-2}$ s the $\eta$ value becomes practically independent of the base doping level in a wide range of $p_{0}$ change. 


\section{A.V. Sachenko et al.: Conversion efficiency in silicon solar cells with...}

At maximum value of doping concentration in the $n^{+}$-region within inter-contact spaces (equal to about $10^{19} \mathrm{~cm}^{-3}$ ) the bulk recombination in the layer with variable donor concentration $N(x)$ is negligible if the $p$ - $n$ junction depth is less than $0.2 \mu \mathrm{m}$, and Shockley-Reed-Hall lifetime in the $n^{+}$-region is more than $10^{-8} \mathrm{~s}$.

\section{References}

1. M.A. Green. Resent advanced in silicon solar cell performance// Proc 10-th European Communities Photovoltaic Solar Energy Conf., Lisbon, p.563(1991)

2. W. Wettling. High efficiency silicon solar cells: State of the art and trends //Solare Energy Materials and Solar Cell. 38, p.487-500 (1995)

3. A.V. Sachenko, N.A. Prima, A.P. Gorban. 1.Efficiency limit for silicon $n^{+}-p-p^{+}$solar cells// Optoelectronika i Poluprovodnikovaya Tekhnika, No.34 (1999)

4. A.P. Gorban, V.P. Kostylev, A.V. Sachenko. Effect of characteristics of front $n^{+}$-layer on the photoconversion efficiency of silicon solar cells//Semiconductor Physics,Quantum Electronics \& Optoelectronics, this issue

5. A. Wang, J. Zhao and M.A. Green. $24 \%$ efficient silicon solar cells // Appl.Phys. Lett. -1990. - 57, №6. -P.602-604.

6. A.L. Fahrenbruch, R.H. Bube. Fundamentals of solar cells. Photovoltaic solar energy conversion,New York,1983
7. A. Ambroziak. Konstruktsiya i tekhnologiya poluprovodnikovykh fotoelektricheskikh priborov (Design and technology of semiconductor photoelectrical devices). Sovetskoe Radio. M., 1970 (in Russian)

8. J. Dziewior and W. Schmid. Auger coefficients for highly doped and highly exited silicon // Appl.Phys.Lett. 31( 5), p.346-348 (1977)

9. A.P. Gorban, V.P. Kostylyov, A.V. Sachenko. The optimization of $n^{+}$ $p-p^{+}$-silicon solar cells parameters. Theoretical relations// Optoelectronika i Poluprovodnikovaya Tekhnika, No.34 (1999) (in Russian)

10. C.H. Wang, K. Misiacos, A. Neugroschel. Minority diffusion lenght in heavity doped silicon // J.Appl.Phys. 54 (22), pp.2233-2234 (1989)

11. P.M. Dunbar, J.R. Hauser. A study of efficiency in low resistivity silicon solar cells// Solid-St. Electron. 19 (2), pp.95-102 (1976)

12. E.K. Banghart, J.L. Gray, R.J. Schwartz. The effects of high level injection on the performance of high intensity, high efficiency silicon solar cells// 20-th IEEE Photovoltaic Spec. Conf., Las Vegas, Sept. 26-30, 1988; Conf. Rec. -New York, 1, pp.717-722 (1988)

13. K. Rajkanan, R. Singh, J. Shewchun . Absorption coefficient of silicon for solar cell // Solid-St.Electron. 22( 9), pp.793-796 (1979)

14. M.B. Spitzer, C.J. Keavney and L.M. Geoffroy. Theoretical and experimental considerations for high silicon solar cell performance // Solar Cells, 17(1), pp.135-149 (1986)

15. H.Y. Fan. Photon-electron interaction, crystals without fields. Berlin, Heidelberg, New-York: Springer-Verlag, 1967. -134p. 